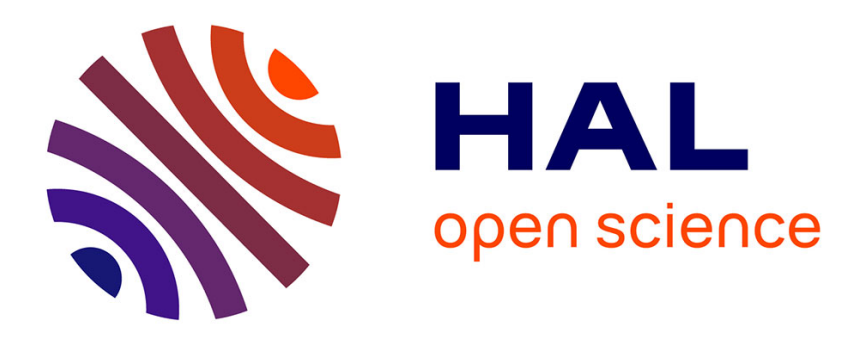

\title{
Multi-relay cooperation with self-energy recycling and power consumption considerations
}

George A Ropokis

\section{To cite this version:}

George A Ropokis. Multi-relay cooperation with self-energy recycling and power consumption considerations. International Workshop on Cooperative Wireless Networks, Oct 2019, Barcelona, Spain. hal-02394899

\section{HAL Id: hal-02394899 \\ https://hal.science/hal-02394899}

Submitted on 5 Dec 2019

HAL is a multi-disciplinary open access archive for the deposit and dissemination of scientific research documents, whether they are published or not. The documents may come from teaching and research institutions in France or abroad, or from public or private research centers.
L'archive ouverte pluridisciplinaire HAL, est destinée au dépôt et à la diffusion de documents scientifiques de niveau recherche, publiés ou non, émanant des établissements d'enseignement et de recherche français ou étrangers, des laboratoires publics ou privés. 


\title{
Multi-relay cooperation with self-energy recycling and power consumption considerations
}

\author{
George A. Ropokis \\ CentraleSupélec/IETR, CentraleSupélec, Campus Rennes, 35510 Cesson Sevigne, France \\ georgios.ropokis@centralesupelec.fr
}

\begin{abstract}
We focus on the problem of rate-optimal beamforming for wireless powered cooperative communications systems. We consider the case of systems employing multiple wireless powered Amplify and Forward self-energy recycling relays and relay selection. By employing a generic piecewise linear energy harvesting model, that can adapt to the characteristics of different non linear energy harvesting measurements and models, we present the solution to the rateoptimal beamformer design problem. Our solution is based on solving a set of simple optimization subproblems where the solution of each one of them can be found in a simple closed form. Moreover, for the first time we combine such a realistic energy harvesting model with an equally realistic power consumption model at the self-energy recycling relays that not only accounts for the transmit power but also for other sources of power consumption (e.g. the power consumption of the relay circuits). By means of simulations we quantify the gains that multiple relays can bring validating that significant gains can be delivered for a variety of relay placements.

Index Terms-Simultaneous Wireless Information and Power Transfer, Amplify and Foward Relaying, Self-energy recycling, Non-linear energy harvesting.
\end{abstract}

\section{INTRODUCTION}

While in conventional Full Duplex (FD) communications the self-interference loop has been recognized as a limiting factor [1], within the paradigm of Simultaneous Wireless Information and Power Transfer (SWIPT), we can find ways to exploit the self-interference loop and increase the energy efficiency of communications systems. Focusing particularly on cooperative communications, the capability of FD relays to simultaneously transmit and receive signals can be employed in order to relay data while harvesting energy. This way, also energy radiated from the relays' own transmit antennas can be harvested, therefore enabling the concept of self-energy recycling in wireless communications. Such approaches have been recently studied in [2]-[5] where system designs for single and multiantenna energy recycling Amplify and Forward (AF) relays were proposed. Moreover, in [6] a new scheme exploiting multiple AF energy recycling relays has been proposed and beamforming solutions for this scheme have been discussed. In addition, more recently communications solutions for energy recycling Detect and Forward relays have also been proposed [7].

While the majority of the above works considers single relay scenarios, it is true that employing multiple wireless powered relays allows for better exploiting the effects of cooperative diversity as well as the potential for wireless
Energy Harvesting (EH) [6]. However, the system design presented in [6] proposes a beamforming scheme based on the simultaneous transmission from the multiple relays, with the beamformer design process itself requiring the use of complicated convex/semidefinite programming methods. As a result, the use of iterative optimization techniques is required such as to solve the resulting beamforming optimization problems and reach near-optimal beamformer designs. More importantly, the algorithmic design presented in [6] is based on an unrealistic linear energy harvesting model, that does not capture the nonlinear characteristics of practical EH circuits, see e.g., [8], as well as on a simple energy consumption model that ignores the energy consumption of the relay circuits. Deviating from such unrealistic assumptions, in this work we study a multi-relay scheme based on relay selection and present the rate-optimal beamforming solution for this scheme accounting for a realistic power consumption model at the relays. Moreover, we combine this model with a flexible piecewise linear EH model that not only allows for accurately modeling the non-linear EH process but also allows for deriving simple closed form expressions for the optimal beamformer, therefore avoiding the use of advanced, iterative optimization techniques and enabling ease of implementation for practical systems. To the best of our knowledge, our work is the first to approach the problem of optimal beamformer design for such cooperative communications systems while adopting realistic models both for the EH process as well as the energy consumption process at the relays.

In brief, the paper's contribution is summarized as follows: 1) We study a communication scheme exploiting multiple self-energy recycling relays and derive the rate-optimal beamformer for it, accounting not only for the nonlinear characteristics of $\mathrm{EH}$, but also for the power consumption characteristics of the relays. 2) By means of simulations we confirm that this scheme can deliver significant performance gains as compared to single relay schemes, for a wide variety of relay placements. Moreover, by means of simulations we identify the positions close to the source of the system as favorable positions for the relays, since they allow for harvesting sufficient amounts of energy such as to power transmissions.

Concerning the presentation of our work, in Section II we present our system model, while in Section III, we solve the problem of optimizing the beamformer design at the selected 
relay, such as to maximize the instantaneous communication rate. In Section IV we evaluate the performance of the proposed scheme for different relay placements and relay availability scenarios. Finally in Section V we present briefly our conclusions as well as some future targets of our research.

Notation: Bold lower case letters are used in order to denote vectors and bold upper case letters are used in order to denote matrices. Operator $(\cdot)^{H}$ stands for the hermitian transpose of a vector/matrix. The $K \times K$ identity matrix is expressed as $\mathbf{I}_{K \times K}$. Notation $\mathbf{w} \sim \mathcal{C N}(\mathbf{0}, \mathbf{R})$ is used in order to denote that random vector $\mathbf{w}$ follows a Complex Gaussian Distribution with a zero mean, and covariance matrix $\mathbf{R}$.

\section{SYSTEM MODEL}

We consider a Single Input Single Output (SISO) source $S$ that communicates with a destination $D$ with the help of $L$ relays, denoted as $R_{l}, l=1, \ldots, L$, each one equipped with $M+1$ antennas. As in [6], [9], each one of the relays is equipped with one rectifier therefore allowing for using one of its antennas for $\mathrm{EH}$ purposes. A slotted communication protocol is adopted where for each time slot one relay is selected to assist $S-D$ communication. Furthermore, we adopt the common assumption that the $S \rightarrow D$ channel is weak enough such that it can be ignored. As a result, during the $t$-th time slot, communication is achieved purely through the $S \rightarrow R_{l^{\star}[t]} \rightarrow D$ channel, where $R_{l^{\star}[t]}$ is the selected relay for the $t$-th time slot, i.e., index $l^{\star}[t]$ indicates the relay selected during time slot $t$. The same time, the remaining, non selected, relays, exploit the transmitted signals for EH purposes. The exact communication protocol is an extension of the communication protocol presented in [9] and [5]. According to it, each time slot that is of duration $T$, is split into two phases, each one of duration $T / 2$. The operation during the two phases is described in what follows, separately for the selected and non selected relays.

\section{A. Operation at the selected relay}

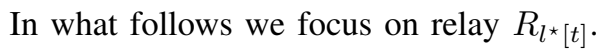

1) Phase I: Data transmission from $S$ : During phase I, $S$ transmits a data signal $x_{s, 1}[t]$ (where $\mathbb{E}\left\{\left|x_{s, 1}\right|^{2}\right\}=1$ ) to relay $R_{l^{\star}[t]}$. Relay $R_{l^{\star}[t]}$ then uses all $M+1$ antennas and applies an MRC combiner $\mathbf{v}[t]$ in order to construct the signal:

$$
y_{r, l^{\star}, 1}[t]=\mathbf{v}^{H}[t]\left(\mathbf{h}_{s r, l^{\star}}[t] \sqrt{P_{s}} x_{s, 1}[t]+\mathbf{n}_{r, l^{\star}, 1}[t]\right) .
$$

In (1), $P_{s}$ stands for the transmit power used by $S, \mathbf{h}_{s r, l^{\star}}[t]$ is the $S \rightarrow R_{l^{\star}[t]}, M+1 \times 1$ Single Input Multiple Output (SIMO) channel, and $\mathbf{n}_{r, l^{\star}, 1}[t] \sim \mathcal{C N}\left(\mathbf{0}, \sigma^{2} \mathbf{I}_{M+1 \times M+1}\right)$, is the Additive White Gaussian Noise (AWGN) at $R_{l^{\star}[t]}$. Since for an MRC combiner it holds that $\mathbf{v}[t]=$ $\mathbf{h}_{s r, l^{\star}}[t] /\left\|\mathbf{h}_{s r, l^{\star}}[t]\right\|$, by introducing the quantity $G_{s r, l^{\star}}[t]=$ $\left\|\mathbf{h}_{s r, l^{\star}}[t]\right\|^{2}$, (1) can also be written as:

$$
y_{r, l^{\star}, 1}[t]=\sqrt{G_{s r, l^{\star}}[t]} \sqrt{P_{s}} x_{s, 1}[t]+\underbrace{\mathbf{v}^{H}[t] \mathbf{n}_{r, l^{\star}, 1}[t]}_{\tilde{n}_{r, l^{\star}, 1}[t]} .
$$

2) Phase II: Data transmission/energy harvesting from/at $R$ : During phase II, $R_{l^{\star}[t]}$ amplifies $y_{r, l^{\star}, 1}[t]$ and exploiting $M$ of its antennas, say antennas $2, \ldots, M+1$, forwards it to $D$. Concurrently, $S$ transmits another signal which is used by the selected relay for energy harvesting purposes. For this purpose, the relay $R_{l}^{\star}[t]$ employs antenna 1 , by connecting it to the rectifier.

Considering first the data forwarding process, assuming an AF protocol, $R_{l^{\star}[t]}$ employs a beamformer $\mathbf{x}[t]$ of power $\|\mathbf{x}[t]\|^{2}=P_{r}[t]$ at its transmits antennas, and forwards the resulting signal, after normalizing it such as to obtain a signal of power $P_{r}[t]$. The signal reaching $D$ is then expressed as:

$$
y_{d}[t]=\frac{\mathbf{x}^{H}[t] \mathbf{h}_{r d, l^{\star}}[t] y_{r, l^{\star}, 1}[t]}{\sqrt{G_{s r, l^{\star}}[t] P_{s}+\sigma^{2}}}+n_{d, 2}[t],
$$

where $\mathbf{h}_{r d, l^{\star}}[t]$ is the $R_{l^{\star}[t]} \rightarrow D$ Multiple Input Single Output (MISO) channel formed between the $M$ transmit antennas of $R_{l^{\star}[t]}$, (i.e., antennas $2, \ldots M+1$ ) and the receive antenna of $D$, and $n_{d, 2}[t] \sim \mathcal{C N}\left(0, \sigma^{2}\right)$ is the AWGN at $D$.

Focusing now on the EH process at $R_{l^{\star}[t]}$, the signal that reaches $R_{l^{\star}[t]}$ during phase II, is expressed as:

$$
\begin{aligned}
y_{r, l^{\star}, 2}[t]= & \sqrt{P_{s}} h_{s r, l^{\star}}[t] x_{s, 2}[t] \\
& +\frac{\mathbf{x}^{H}[t] \mathbf{h}_{l o o p} y_{r, l^{\star}, 1}[t]}{\sqrt{G_{s r, l^{\star}}[t] P_{s}+\sigma^{2}}}+n_{r, l^{\star}, 2}[t],
\end{aligned}
$$

where $h_{s r, l^{\star}}[t]$ is the channel formed between $S$ and the harvesting antenna of $R_{l^{\star}[t]}$, and $x_{s, 2}[t]$ is the signal transmitted by $S$ for $\mathrm{EH}$ purposes. Moreover, $\mathbf{h}_{l o o p}$ characterizes the MISO channel formed between the transmit antennas of $R_{l^{\star}[t]}$ and its EH antenna. In what follows, we assume that $\mathbf{h}_{\text {loop }}$ is deterministic and identical for all relays.

As explained earlier, the signal $y_{r, l^{\star}, 2}[t]$ in (4) is exploited at $R_{l^{\star}[t]}$ for EH purposes. For the EH process we consider a piecewise linear model. According to it, if the $\mathrm{EH}$ circuit is fed with a signal $x[t]$ of average power $P_{i n}=\mathbb{E}\left\{|x[t]|^{2}\right\}$, the harvested power is related to $P_{i n}$ through the expression:

$P_{h}\left(P_{\text {in }}\right)=\eta_{i} P_{\text {in }}+\mu_{i}, \quad P_{\text {in }} \in\left[P_{\text {low }, i}, P_{\text {up }, i}\right], i=1, \ldots, I$,

where $\eta_{i}, \mu_{i}$, are positive parameters related to the $\mathrm{EH}$ circuit, and $P_{l o w, i}, P_{u p, i}$, are the lower and upper points respectively for the $i$-th segment of the EH model. As we will see in later sections, such a model allows for closely approximating several other composite EH models, such as the accurate models discussed in [8]. Moreover, it encompasses as a special case the saturated model of [10], that is the simplest model capturing the non-linear characteristics of EH circuits. Finally, we also note that such piecewise linear models have also been recently proposed in [11] for the purposes of the performance analysis of wireless powered communications systems.

As in [9], we select signal $x_{s, 2}$ as $x_{s, 2}[t]=x_{s, 1}[t] e^{j \theta[t]}$, where $\theta[t]$ is the phase of $\mathbf{x}^{H}[t] \mathbf{h}_{\text {loop }}$. As a result, assuming that the noise power is small enough such that the noise contribution to $\mathrm{EH}$ can be ignored, it can be shown that the 
energy harvested by the relay comes from the transmissions of $S$ and $R_{l^{\star}[t]}$ itself and is given as:

$$
\begin{aligned}
& e_{W, l^{\star}}[t]= \\
& \frac{T P_{h}\left(P_{s}\left(\sqrt{g_{s r, l^{\star}}[t]}+\frac{\sqrt{G_{s r, l^{\star}}[t]}\left|\mathbf{x}^{H}[t] \mathbf{h}_{\text {loop }}\right|}{\sqrt{G_{s r, l^{\star}}[t] P_{s}+\sigma^{2}}}\right)^{2}\right)}{2},
\end{aligned}
$$

where for the ease of presentation we have introduced the notation $g_{s r, l^{\star}}[t]=\left|h_{s r, l^{\star}}[t]\right|^{2}$. As explained in [9], where a battery architecture for systems that simultaneously transmit and harvest energy is discussed, the amount of energy in (6) harvested during phase II, only becomes available for consumption to $R_{l^{\star}[t]}$, at the end of the $t$-th time slot. Therefore this amount of energy is not directly available for use during timeslot $t$. However, in what follows we introduce a system design that accounts for knowledge that this amount of energy will become available at the end of the timeslot. In more detail, we consider a system design that operates based on the following two energy consumption constraints, which are similar to the constraints presented in [6].

Energy preservation constraint: We assume that the power level $P_{r}[t]$ is selected such that the energy available at $R_{l^{\star}[t]}$ at the end of the $t$-th timeslot, hereby denoted as $E_{l^{\star}[t]}[t], t \geq 1$, is at least equal to the amount of energy $E_{l^{\star}[t]}[0]$ available to it at the beginning of transmission. By adopting this constraint, it is ensured that none of the relays consumes its own energy resources at any time instance and as a result, after the completion of transmission, the energy budget of the relays has not been impacted by their use for communication.

In order to mathematically express this constraint we start by defining the energy surplus available at relay $R_{l^{\star}}[t]$ at the beginning of timeslot $t$ as the difference between the energy available to the relay at the beginning of time instance $t$, and the energy level $E_{l^{\star}}[0]$, i.e., as:

$$
e_{s, l^{\star}}[t]=E_{l^{\star}[t]}[t-1]-E_{l^{\star}[t]}[0] .
$$

Such an energy surplus may appear in case that some time slots are devoted purely for EH purposes due to the fact that another relay had been selected for transmission.

As a further step in order to quantify the energy preservation constraint, we now also introduce our power consumption model at the relay. In more detail we base our model on the energy consumption model presented in [12] that captures both the energy consumption during data reception (i.e. during phase I of the protocol ) and during transmission (i.e. during phase II of the protocol). According to it, the energy consumed by relay $R_{l^{\star}}[t]$ during a timeslot, is expressed as

$$
E_{\text {cons }}=P_{C, r_{x}} \frac{T}{2}+\left(\frac{P_{r}[t]}{\varepsilon}+P_{C, t_{x}}\right) T / 2 .
$$

In the above equation, the term $P_{C, r_{x}}$ corresponds to the power consumption of the receive circuits of the selected relay, which are used for the first $T / 2$ seconds of the timeslot. This term captures the power consumption of the $M+1$ receive $\mathrm{RF}$ chains associated with the antennas of the selected relay.
On the other hand, focusing on the remaining terms of (8), the term $P_{C, t_{x}}$ corresponds to the power consumption of the transmit circuits, which are used for the $T / 2$ seconds of the second phase of the timeslot. This term captures the power consumption of the $M$ transmit RF chains associated with each one of the transmit antennas of the relay. Finally, we assume that each one of the transmit RF chains is connected to a power amplifier, characterized by a linear input-output power consumption formula. As a result, the actual power consumed by the power amplifiers for a transmission of total transmit power $P_{r}[t]$ is given as $P_{r}[t] / \varepsilon$, where $\varepsilon \leq 1$ denotes the common (for all amplifiers) efficiency.

Accounting for this power consumption model, the energy preservation constraint is finally expressed as:

$$
P_{C, r_{x}} \frac{T}{2}+\left(\frac{P_{r}[t]}{\varepsilon}+P_{C, t_{x}}\right) T / 2 \leq e_{W, l^{\star}}[t]+e_{s, l^{\star}}[t],
$$

or equivalently, after introducing the term $P_{C}=P_{C, t_{x}}+P_{C, r_{x}}$, as:

$$
\left(\frac{P_{r}[t]}{\varepsilon}+P_{C}\right) T / 2 \leq e_{W, l^{\star}}[t]+e_{s, l^{\star}}[t] .
$$

Using (10), it is then straightforward to show that the energy $E_{l^{\star}[t]}[t]$ available at $R_{l^{\star}[t]}$ at the end of the $t$-th timeslot is equal to:

$$
E_{l^{\star}[t]}[t]=E_{l^{\star}[t]}[0]+e_{s, l^{\star}}[t]+e_{W, l^{\star}}[t]-\frac{\left(\frac{P_{r}[t]}{\varepsilon}+P_{C}\right) T}{2}
$$

and is indeed greater than (or equal to) $E_{l^{\star}[t]}[0]$.

Energy causality constraint: We further introduce the constraint that the energy consumed during the $t$-th timeslot is at most equal to the amount of energy available at the battery at the beginning of timeslot $t$, or equivalently at the end of timeslot $t-1$. As a result, we have that

$$
\left(\frac{1}{\varepsilon} P_{r}[t]+P_{C}\right) T / 2 \leq E_{l^{\star}[t]}[t-1]=E_{l^{\star}[t]}[0]+e_{s, l^{\star}}[t] .
$$

Finally, we note that combining the energy causality and preservation constraints of (10) and (12) with the energy harvesting model in (5), we can express both constraints in the form of (C1.1) and (C1.2), for each one of the $I$ intervals of the EH model.

\section{B. Operation at the remaining relays}

We now describe the mode of operation for each one of the non selected relays, i.e., for relays $R_{l}, l \neq l^{\star}[t]$. For this purpose, we investigate separately the two phases of the communication protocol.

1) Phase I: Harvesting energy from the signal transmitted by $S$ : During phase I, $R_{l}$ uses the antenna connected to the rectifier, i.e., antenna 1 , to harvest energy from the signal arriving at it. One can write this signal in the form of the following equation: 


$$
\begin{gathered}
\|\mathbf{x}[t]\|^{2} \leq \min \left\{\eta_{i} \varepsilon\left(\sqrt{g_{s r, l^{\star}}[t] P_{s}}+\frac{\sqrt{G_{s r, l^{*}}[t] P_{s}}\left|\mathbf{x}^{H}[t] \mathbf{h}_{l o o p}\right|}{\sqrt{G_{s r, l^{\star}}[t] P_{s}+\sigma^{2}}}\right)^{2}+\varepsilon \mu_{i}+\frac{2 \varepsilon e_{s, l^{\star}}[t]}{T}, \frac{2 \varepsilon E_{l^{\star}[t]}[t-1]}{T}\right\}-\varepsilon P_{C} \\
P_{l o w, i} \leq\left(\sqrt{g_{s r, l^{\star}}[t] P_{s}}+\sqrt{\frac{G_{s r, l^{\star}}[t] P_{s}}{G_{s r, l^{\star}}[t] P_{s}+\sigma^{2}}}\left|\mathbf{x}^{H}[t] \mathbf{h}_{\text {loop }}\right|\right)^{2} \leq P_{u p, i}, \quad i=1, \ldots, I .
\end{gathered}
$$

$$
y_{r, l, 1}[t]=h_{s r, l}[t] \sqrt{P_{s}} x_{s, 1}[t]+n_{r, l, 1}[t] .
$$

In (13), $h_{s r, l}[t]$ is the channel between $S$ and the EH antenna of $l$ and $n_{r, l, 1}[t] \sim \mathcal{C N}\left(0, \sigma^{2}\right)$ is the AWGN at $R_{l}$. Assuming again that noise power is substantially lower than the power of the transmit signal, based on (5), the amount of energy that can be harvested from signal $y_{r, l, 1}[t]$ is given as:

$$
e_{w, l, 1}[t]=T P_{h}\left(\left|h_{s r, l}[t]\right|^{2} P_{s}\right) / 2 .
$$

2) Phase II: Harvesting energy from the signals transmitted by $S$ and $R_{l^{\star}}[t]$ : During the second phase, relay $R_{l}, l \neq$ $l^{\star}[t]$ again harvests the energy of the signal reaching it's EH antenna. This signal can be expressed as:

$$
\begin{aligned}
& y_{r, l, 2}[t]=h_{s r, l}[t] \sqrt{P_{s}} x_{s, 1}[t] e^{j \theta[t]} \\
& +\mathbf{x}^{H}[t] \mathbf{h}_{l^{\star}, l}[t] \frac{\sqrt{G_{s r, l^{\star}}[t] P_{s}} x_{s, 1}+\tilde{n}_{r, l^{\star}, 1}[t]}{\sqrt{G_{s r, l^{*}}[t] P_{s}+\sigma^{2}}}+n_{r, l, 2}[t],
\end{aligned}
$$

where $\mathbf{h}_{l^{\star}, l}[t]$ stands for the channel formed between the $M$ transmit antennas of relay $R_{l^{\star}[t]}$ and the EH antenna of $R_{l}$. As a result, based again on the assumption that noise power is small enough, the energy that can be harvested can be proven to be equal to [6]:

$$
e_{w, l, 2}[t]=\frac{T P_{h}\left(P_{s}\left|h_{s r, l}[t] e^{j \theta[t]}+\frac{\mathbf{x}^{H}[t] \mathbf{h}_{l^{\star}, l}[t] \sqrt{G_{s r, l}[t]}}{\sqrt{G_{s r, l \star}[t] P_{s}+\sigma^{2}}}\right|^{2}\right)}{2} .
$$

Hence, the energy available at relay $R_{l}$ at the end of time instance $t$ is equal to:

$$
E_{l}[t]=E_{l}[t-1]+e_{w, l, 1}[t]+e_{w, l, 2}[t] .
$$

\section{THE OPTIMAL BEAMFORMING PROBLEM}

The focus in this work is on maximizing the instantaneous rate, or equivalently, due to the monotonic relationship between the instantaneous rate and the SNR, on maximizing the instantaneous SNR $\rho[t]$. Given the form of the received signal at $D$, the following convenient form can be derived for $\rho[t]:$

$$
\rho[t]=\frac{1}{\sigma^{2}} \frac{G_{s r, l^{\star}}[t] P_{s}[t]\left|\mathbf{x}[t]^{H} \mathbf{h}_{r d, l^{\star}}[t]\right|^{2}}{G_{s r, l^{\star}}[t] P_{s}[t]+\left|\mathbf{x}[t]^{H} \mathbf{h}_{r d, l^{\star}}[t]\right|^{2}+\sigma^{2}} .
$$

As a result, exploiting also constraints (C1.1) and (C1.2), the problem of rate-optimal beamforming boils down to solving the subproblems:

$$
\underset{\mathbf{x}[t]}{\operatorname{maximize}:} \rho[t] \text { subject to: }(\mathrm{C} 1.1),(\mathrm{C} 1.2),
$$

for each one of the $I$ intervals of the form $\left[P_{l o w, i}, P_{u p, i}\right]$, and selecting the subproblem solution that results in the maximum SNR. Before continuing with solving (P1), for the purposes of simplifying the presentation, in what follows we drop the dependency of $\mathbf{x}[t]$ as well as of all channel realizations on time. We then note that since the SNR is an increasing function of $\left|\mathbf{x}^{H} \mathbf{h}_{r d, l^{\star}}\right|$, solving (P1) such as to maximize the SNR is equivalent to solving subproblem

$$
\underset{\mathbf{x}}{\operatorname{maximize}:}\left|\mathbf{x}^{H} \mathbf{h}_{r d, l^{\star}}\right| \text { subject to: (C1.1), (C1.2), }
$$

for each one of the $I$ intervals of the EH model. In order to solve (P2), and therefore subproblem (P1), the following Lemma will be useful.

Lemma 1: A beamformer for solving problem (P2) can be found in the form:

$$
\mathbf{x}=\gamma_{1} e^{j \phi_{1}} \mathbf{u}_{1}+\gamma_{2} e^{j \phi_{2}} \mathbf{u}_{2}
$$

where $\mathbf{u}_{1}=\frac{\mathbf{h}_{\text {loop }}}{\left\|\mathbf{h}_{\text {loop }}\right\|}, \quad \mathbf{u}_{2}=\frac{\left(\mathbf{I}-\mathbf{u}_{1} \mathbf{u}_{1}^{H}\right) \mathbf{h}_{r d, l^{\star}}}{\left\|\left(\mathbf{I}-\mathbf{u}_{1} \mathbf{u}_{1}^{H}\right) \mathbf{h}_{r d, l^{\star}}\right\|}$,

and $\gamma_{i} \in \mathbb{R}^{+}, i=1,2$ and $\phi_{i} \in[0,2 \pi), i=1,2$.

Proof: Following an approach similar to the one in [4], we start by considering an orthonormal basis $\left\{\mathbf{u}_{1}, \ldots, \mathbf{u}_{N_{R}}\right\}$ for $\mathcal{C}^{M \times 1}$, with $\mathbf{u}_{1}$ and $\mathbf{u}_{2}$ defined as in (22). Using this orthonormal basis, we can write any beamformer in the form:

$$
\mathbf{x}=\sum_{i=1}^{M} \gamma_{i} e^{j \phi_{i}} \mathbf{u}_{i}
$$

The objective function in (P2) is then expressed as

$$
\left|\mathbf{x}^{H} \mathbf{h}_{r d, l^{\star}}\right|=\left|\left(\gamma_{1} e^{j \phi_{1}} \mathbf{u}_{1}+\gamma_{2} e^{j \phi_{2}} \mathbf{u}_{2}\right)^{H} \mathbf{h}_{r d, l^{\star}}\right|,
$$

while (C1.1) and (C1.2) can also be simplified by noticing that

$$
\left|\mathbf{x}^{H} \mathbf{h}_{\text {loop }}\right|=\gamma_{1}\left|\mathbf{u}_{1}^{H} \mathbf{h}_{\text {loop }}\right|, \text { and }\|\mathbf{x}\|^{2}=\sum_{i=1}^{N} \gamma_{i}^{2} .
$$

As a result, based on (24), we see that values of coefficients $\gamma_{i}, i \geq 3$ do not influence the utility function value of (P2). On the other hand, based on (25), we see that non zero values of 
$\gamma_{i}, i \geq 3$, restrict the feasible values of $\gamma_{1}$ and $\gamma_{2}$. Therefore, it is straightforward to show that the optimal solution to (P2) is obtained for $\gamma_{3}, \ldots, \gamma_{N}=0$.

Building on the above Lemma, the following theorem further confines the form of the optimal beamformer.

Theorem 1: The optimal solution of (P1) and (P2) is given as:

$$
\mathbf{x}=\gamma_{1} e^{j \phi_{1}} \mathbf{u}_{1}+\gamma_{2} e^{j \phi_{2}} \mathbf{u}_{2}
$$

where $\phi_{1}, \phi_{2} \in[0,2 \pi)$ are selected such that

$$
\frac{e^{-j \phi_{1}} \mathbf{u}_{1}^{H} \mathbf{h}_{r d, l^{\star}}}{\left|\mathbf{u}_{1}^{H} \mathbf{h}_{r d, l^{\star}}\right|}=\frac{e^{-j \phi_{2}} \mathbf{u}_{2}^{H} \mathbf{h}_{r d, l^{\star}}}{\left|\mathbf{u}_{2}^{H} \mathbf{h}_{r d, l^{\star}}\right|}
$$

and $\gamma_{1}, \gamma_{2}$ are found to be the solution to problem (P3).

Proof: Using the result of Lemma 1 and restricting ourselves to beamformers of the form (21), it follows that the value obtained for the utility function in (P2) is given as in (24), where this value is maximized if terms $e^{-j \phi_{1}} \mathbf{u}_{1}^{H} \mathbf{h}_{r d, l^{*}}$ and $e^{-j \phi_{2}} \mathbf{u}_{2}^{H} \mathbf{h}_{r d, l^{\star}}$, are of the same phase, i.e., if property (27) holds. Moreover, based on (25), we can see that for a beamformer of the form (21), the choice of phase parameters $\phi_{1}, \phi_{2}$, does not affect the feasibility of the beamfomer. Hence, the optimal beamformer can be found by selecting phase values $\phi_{1}$ and $\phi_{2}$ such as to satisfy property (27). With this choice, we then have that:

$$
\left|\mathbf{x}^{H} \mathbf{h}_{r d, l^{\star}}\right|=\gamma_{1}\left|\mathbf{u}_{1}^{H} \mathbf{h}_{r d, l^{\star}}\right|+\gamma_{2}\left|\mathbf{u}_{2}^{H} \mathbf{h}_{r d, l^{\star}}\right|,
$$

and the optimal beamformer problem boils down to determining the values of $\gamma_{1}$ and $\gamma_{2}$ such as to maximize the value of the objective function in (28).

Combining equations (28) and (25) we then see that solving subproblem (P1) reduces to solving the problem (P3):

$$
\begin{array}{cl}
\operatorname{maximize}: & \alpha \gamma_{1}+\beta \gamma_{2} \\
\text { subject to: } & \gamma_{1}^{2}+\gamma_{2}^{2} \leq\left(A_{i}+B_{i} \gamma_{1}\right)^{2}+C_{i}, \\
& \gamma_{1}^{2}+\gamma_{2}^{2} \leq D, \\
& \gamma_{1, \text { low }, i} \leq \gamma_{1} \leq \gamma_{1, \text { up }, i}, \quad \gamma_{2} \in \mathbb{R}^{+}
\end{array}
$$

where $\alpha=\left|\mathbf{u}_{1}^{H} \mathbf{h}_{r d, l^{\star}}\right|, \beta=\left|\mathbf{u}_{2}^{H} \mathbf{h}_{r d, l^{\star}}\right|$,

$A_{i}=\sqrt{\eta_{i} \varepsilon g_{s r, l^{\star}} P_{s}}, \quad B_{i}=\sqrt{\frac{\eta_{i} \varepsilon G_{s r, l^{\star}} P_{s}}{G_{s r, l^{\star}} P_{s}+\sigma^{2}}}\left|\mathbf{u}_{1}^{H} \mathbf{h}_{l o o p}\right|$,

$C_{i}=\mu_{i} \varepsilon+\frac{2 \varepsilon e_{s, l^{\star}[t]}[t]}{T}-\varepsilon P_{C}, D=\frac{2 \varepsilon E_{l^{\star}[t]}[t-1]}{T}-\varepsilon P_{C}$,

$\gamma_{1, \text { low }, i}=\max \left\{0, \frac{\sqrt{\frac{G_{s r, l^{\star} P_{s}+\sigma^{2}}}{G_{s r, l^{\star}} P_{s}}}\left(\sqrt{P_{l o w, i}}-\sqrt{g_{s r, l^{\star} P_{s}}}\right)}{\left|\mathbf{u}_{1}^{H} \mathbf{h}_{\text {loop }}\right|}\right\}$

and

$$
\gamma_{u p, i}=\max \left\{0, \frac{\sqrt{\frac{G_{s r, l^{\star} P_{s}+\sigma^{2}}}{G_{s r, l^{\star}} P_{s}}}\left(\sqrt{P_{u p, i}}-\sqrt{g_{s r, l^{\star} P_{s}}}\right)}{\left|\mathbf{u}_{1}^{H} \mathbf{h}_{l o o p}\right|}\right\} .
$$

Hence, the result of Theorem 1 is proven.

In (P3), constraints (C3.1) and (C3.2) provide an equivalent formulation of $(\mathrm{C} 1.1)$, while $(\mathrm{C} 3.3)$ corresponds to $(\mathrm{C} 1.2)$. Moreover, constraint $\gamma_{2} \in \mathbb{R}^{+}$can be ignored, since positive values of $\gamma_{2}$ result in higher values for the the utility function in (P3) (with respect to negative values), while the sign of $\gamma_{2}$ does not affect the feasibility of the remaining constraints of (P3). Taking this into account, in the appendix we present our approach for solving (P3). Moreover, we highlight here that the approach followed in the appendix for solving (P3), results in solving a set of simple subproblems where for each one of them, the solution can be expressed in closed form using simple, analytical functions. As a result, the use of advanced optimization techniques, e.g. iterative optimization techniques is avoided, allowing for the ease of implementation of the proposed beamforming scheme.

Finally, the following theorem discusses some additional properties of problems (P1) and (P3), that are exploited in the solution process.

Theorem 2: For the optimal beamformer at least one of constraints (C3.1) and (C3.2) is active.

Proof: Let us denote as $\hat{\gamma}_{1}, \hat{\gamma}_{2}, \hat{\gamma}_{2} \geq 0$, an optimal solution for (P3) for which both constraints (C3.1) and (C3.2) are inactive. Since the right hand sides of (C3.1), (C3.2) do not vary with $\gamma_{2}$, and (C3.3) does not depend on $\gamma_{2}$, it then holds that another feasible point $\left(\hat{\gamma}_{1}, \tilde{\gamma}_{2}\right)$, with $\tilde{\gamma}_{2} \geq \hat{\gamma}_{2}$ selected such that $\hat{\gamma}_{1}^{2}+\tilde{\gamma}_{2}^{2}=\min \left\{\left(A_{i}+B_{i} \hat{\gamma}_{i}\right)^{2}+C, D\right\}$ can be found that clearly satisfies one of constraints (C3.1), (C3.2) with equality. Nevertheless, it is obvious that such a point, since $\tilde{\gamma}_{2} \geq \hat{\gamma}_{2}$, results in a higher value for the utility function of (P3). Hence, no point that results in both constraints (C3.1) and (C3.2) being inactive can be an optimal point. Thus the result of Theorem 2 is proven.

Having presented the solution for the beamforming problem in the following section we present results obtained by using the proposed scheme.

\section{NUMERICAL RESULTS AND DISCUSSION}

We first present the considered simulation scenario, starting from the considered energy harvesting model.

\section{A. The considered energy harvesting model}

We adopt a piecewise linear approximation of the following highly accurate EH model proposed in [8]:

$$
P_{h}^{a c c}=\frac{\left(a-\frac{b}{c}\right) P_{i n}}{P_{i n}+c},
$$

where $P_{h}^{a c c}$ is the harvested power, $P_{\text {in }}$ is the input power to the harvesting circuit (measured in mWatts), and parameters $a, b$, and $c$ are selected to fit real $\mathrm{EH}$ measurements. Following [8] we set $a=2.463, b=1.635$, and $c=0.826$.

As a result of the selection of the specific parameter values, function $P_{h}^{a c c}\left(P_{i n}\right)$ is a concave function of $P_{i n}$. Therefore, for any interval of the form $\left[P_{l o w, i}, P_{u p, i}\right]$, the inequality (34) is satisfied. 


$$
P_{h}^{a c c} \geq \eta_{i} P_{\text {in }}+\mu_{i}, \quad \forall P_{\text {in }} \in\left[P_{\text {low }, i}, P_{u p, i}\right], \text { where } \eta_{i}=\frac{P_{h}^{a c c}\left(P_{u p, i}\right)-P_{h}^{a c c}\left(P_{l o w, i}\right)}{P_{u p, i}-P_{\text {low }, i}}, \text { and } \mu_{i}=P_{h}^{a c c}\left(P_{l o w, i}\right)-\eta_{i} P_{l o w, i}
$$

Based on the EH expression in (33), we model the EH process using (5), with $\eta_{i}$ and $\mu_{i}$ defined in (34). Following such an approach, and by selecting $P_{\text {low }, i}$ and $P_{u p, i}$ by uniformly splitting the interval $[0,1 \mathrm{mWatts}]$ in ten intervals, and each one of the intervals $[1,5 \mathrm{mWatts}]$ and $[5,20 \mathrm{mWatts}]$ in five intervals a piecewise linear model of the form (5) was obtained. We also note that for values of $P_{\text {in }}$ larger than $20 \mathrm{mWatts}$, we consider that a saturation effect take place. As a result the harvested power is the same for all values of $P_{i n}$ that are higher than (or equal to) $20 \mathrm{mWatts}$. While at a first glance such an approach may lead to loss of accuracy with respect to the exact model in (33), in practice, due to the concave nature of the energy harvesting model in (33), the loss of accuracy caused by this assumption was found to be practically negligible. Indicatively, we note that the accuracy achieved by this piecewise model had a relative approximation error (with respect (33)) that was at most 2.5 $\% \forall P_{\text {in }} \in[0,100 \mathrm{mWatts}]$, therefore allowing to accurately model harvesting, with a small number of intervals, i.e., 21 intervals. We finally highlight that in the presence of pathloss and fading, the input power $P_{i n}$ to the EH circuit is drastically reduced. As a result, for most practical purposes, only a subset of these 21 intervals, i.e. the 10 intervals corresponding to the range $[0,1 \mathrm{mWatt}]$, need to be considered. Hence, the complexity is reduced.

\section{B. The considered channel model and simulation scenario}

We study a cooperative communications system where the distance $d_{s d}$ between the Source $S$ and the destination $D$ is set to be $d_{s d}=30 \mathrm{~m}$. We set $S$ to be at the origin of our coordinate system and we place $D$ at coordinates $\left(d_{s d}, 0\right)$. Moreover, we consider a system with two relays $R_{1}$ and $R_{2}$ positioned symmetrically at coordinates $\left(\frac{d_{s d}}{2}, \frac{d_{s d}}{4}\right)$ and $\left(\frac{d_{s d}}{2},-\frac{d_{s d}}{4}\right)$. To model the wireless channel, we use pathloss and i.i.d. block Rayleigh fading. Hence, for a relay $R_{l}$ positioned at distance $d_{s r, l}$ from $S$ and $d_{r d, l}$ from $D$, it holds that the pathloss for the $S \rightarrow R_{l}$ and $R_{l} \rightarrow D$ channels are expressed as:

$$
\bar{g}_{s r, l}=\frac{\mathcal{G}_{t} \mathcal{A}}{\left(d_{s r, l} / d_{0}\right)^{\epsilon}}, \quad \text { and } \quad \bar{g}_{r d, l}=\frac{\mathcal{G}_{t} \mathcal{A}}{\left(d_{r d, l} / d_{0}\right)^{\epsilon}},
$$

where $\mathcal{G}_{t}$ is the transmit antenna gain that we assume to be common for both $S$ and $R$. Moreover, $\mathcal{A}$ is the pathloss at a nominal distance $d_{0}$. Following the channel model presented in [13], we set the pathloss exponent equal to $\epsilon=2.5$, and attenuation $\mathcal{A}$, corresponding to a distance of $d_{0}=1 \mathrm{~m}$, equal to a value of $-30[d B]$. We also set $\mathcal{G}_{t}$ to be equal to $10[d B]$. The actual communication channels between $S \rightarrow R_{l}$ and $R_{l} \rightarrow D$ are then expressed as:

$$
\mathbf{h}_{s r, l}[t]=\sqrt{\bar{g}_{s r, l}} \tilde{\mathbf{h}}_{s r, l}[t] \text {, and } \mathbf{h}_{r d, l}[t]=\sqrt{\bar{g}_{r d, l}} \tilde{\mathbf{h}}_{r d, l}[t]
$$

with $\tilde{\mathbf{h}}_{s r, l}[t] \sim \mathcal{C N}\left(\mathbf{0}, \mathbf{I}_{M+1 \times M+1}\right)$, and similarly $\tilde{\mathbf{h}}_{r d, l}[t] \sim$ $\mathcal{C N}\left(\mathbf{0}, \mathbf{I}_{M \times M}\right)$.

We follow a similar block Rayleigh fading approach for modeling the channel formed between the transmit antennas of relay $l_{1}, l_{1}=1 \ldots, L$, and the energy harvesting antenna of relay $l_{2}, l_{2}=1, \ldots, L$, with $l_{1} \neq l_{2}$. In more detail, we consider that the $R_{l_{1}} \rightarrow R_{l_{2}}$ channel is of the form:

$$
\mathbf{h}_{l_{1}, l_{2}}[t]=\sqrt{\bar{g}_{l_{1}, l_{2}}} \tilde{\mathbf{h}}_{l_{1}, l_{2}}[t], \text { with } \quad \bar{g}_{l_{1}, l_{2}}=\frac{\mathcal{G}_{t} \mathcal{A}}{\left(d_{l_{1}, l_{2}} / d_{0}\right)^{\epsilon}}
$$

where $d_{l_{1}, l_{2}}$ is the distance between relay $R_{l_{1}}$ and relay $R_{l_{2}}$, and $\tilde{\mathbf{h}}_{l_{1}, l_{2}}[t] \sim \mathcal{C N}\left(\mathbf{0}, \mathbf{I}_{M \times M}\right)$. Concerning the selfenergy recycling loop channel $\mathbf{h}_{\text {loop }}$, we set it equal to $\mathbf{h}_{\text {loop }}=\sqrt{\delta} \mathbf{1}_{M \times 1}$ where $\delta=-15[d B]$, as in [9]. Moreover, we set the number of antennas at the relays to be equal to $M+1=5$ and the noise power to $\sigma^{2}=-90[\mathrm{dBm}]$ [13]. Finally, the time slot duration $T$ was set to $T=1$ second.

Based on the above simulation parameters, in Figure 1 we present the performance of the proposed beamforming scheme, measured in terms of the average (over $10^{4}$ channel realizations) achievable rate, as a function of transmit power $P_{s}$. Concerning the additional power consumption $P_{C}$ of the relay, we base our selection of value on recent results related to the design of low power transceivers for EH applications (e.g. [14], [15]) that propose transceiver designs characterized by power consumption of the order of tens of $\mu$ Watts. With this in mind, we set $P_{C}=50 \mu W$ atts. Concerning relay selection, we assume that for each time instance, the relay resulting in the maximum instantaneous SNR is selected. Finally, we mention that in Fig. 1, for the purposes of comparison, we also include results corresponding to the case that only relay $R_{1}$ is available and again the proposed beamforming scheme is used, as well as results corresponding to the case that an additional relay $R_{3}$ is also available. We place this additional relay in close proximity to $R_{2}$ (i.e., at coordinates $\left(\frac{d_{s d}}{2},-\frac{d_{s d}}{4}+1 \mathrm{~m}\right)$ ) such that we can consider that the effects of pathloss are nearly identical for all three relays. We finally note, that in case of an infeasibility in the optimal beamforming problem for all available relays, we exploit the corresponding time slot for the purposes of energy harvesting, at all available relays. As we can see in Figure 1, the addition of the second and third relays results in significant performance improvement. Indicatively, for a value of $P_{s}$ equal to $30 \mathrm{dBm}$, the addition of $R_{2}$ results in a performance improvement of nearly $100 \%$, doubling the transmit rate (with respect to the single relay case) while the addition of $R_{3}$ results in a performance improvement of nearly $200 \%$ practically tripling the rate (with respect to the single relay case).

As a second test, we now investigate the effect of relay 


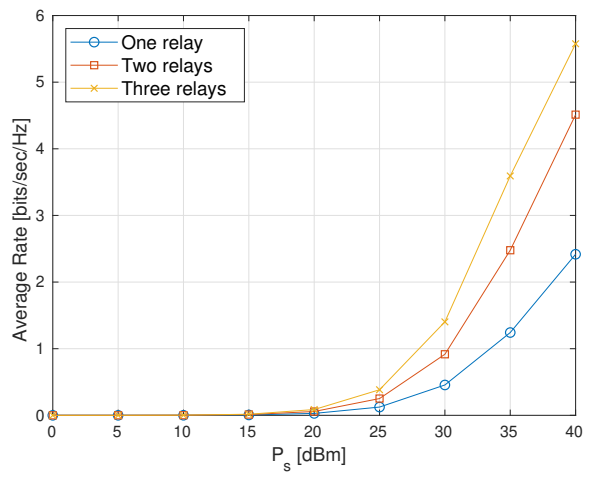

Fig. 1. The transmit rate as a function of $P_{s}$ for different numbers of relays.

placement on the benefits that relay selection can deliver. To this end, we consider a system with two relays namely $R_{1}$ and $R_{2}$ and as in the previous case, we place relay $R_{1}$ at coordinates $\left(d_{s d} / 2, d_{s d} / 4\right)$. On the other hand, for relay $R_{2}$ we consider placements of the form $\left(d_{s d} / 2,-y\right)$ and set different values for $y$. Finally, concerning the transmit power $P_{s}$ we investigate the case that $P_{s}$ is selected to be equal to $30[\mathrm{dBm}]$ as well as the case that $P_{s}=25[\mathrm{dBm}]^{1}$. For the two above values of $P_{s}$, in Fig. 2 we present the relative performance gain that relay $R_{2}$ delivers (with respect to the case that only $R_{1}$ is present) as a function of the normalized distance $y / d_{s d}$. We measure this performance gain as:

$$
\text { Performance Gain }=\left(\mathcal{C}_{1,2}-\mathcal{C}_{1}\right) / \mathcal{C}_{1} \times 100 \%,
$$

where $\mathcal{C}_{1}$ stands for the average rate in case that only $R_{1}$ is available, and $\mathcal{C}_{1,2}$ for the average rate in case that both $R_{1}$ and $R_{2}$ are available. We observe that for both values of $P_{s}$, a very similar trend for the performance gain as a function of $y$ appears. Moreover, for values of $y$ less than $d_{s d} / 2$ a performance gain of at least nearly $50 \%$ is achieved, indicating that significant gains can be delivered for several relay placements. On the other hand, for $y>d_{s d}$ the performance gains delivered by the additional relay nearly vanish.

Finally, we further investigate the effect of relay positioning by considering the case of two available relays and placements of the form $\left(x, d_{s d} / 4\right)$ for relay $R_{1}$ and $\left(x,-d_{s d} / 4\right)$ for relay $R_{2}$. For such placements, after setting $P_{s}=30[\mathrm{dBm}]$, in Fig. 3 we plot the achievable rate as a function of the normalized distance $x / d_{s d}$. The remaining system parameters were once more set to the values specified earlier. Based on the results presented in Fig. 3, we observe that placements of the two relays closer to $S$ result in higher achievable rate. This implies that the achievable rate is mostly influenced by the ability of the relays to harvest sufficient energy for their transmissions.

\section{CONCLUSIONS AND FUTURE WORK}

We have presented a multi-relay scheme employing selfenergy recycling relays and we have developed a new

\footnotetext{
${ }^{1}$ The remaining simulation parameters are set to the values specified earlier.
}

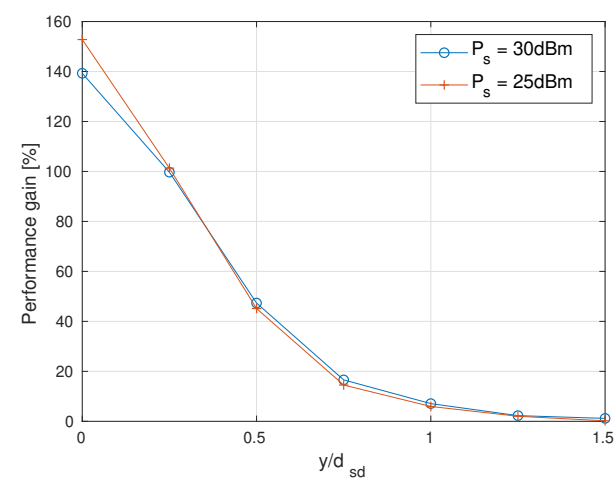

Fig. 2. Performance gain delivered by the second relay as a function of $y$.

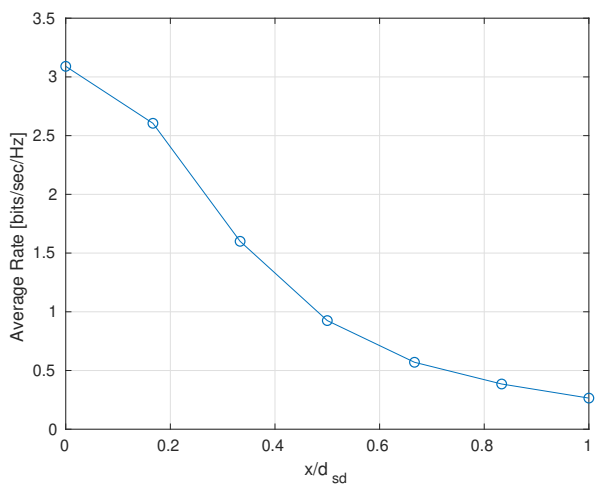

Fig. 3. Achievable average rate as a function of $x$, for $P_{s}=30 \mathrm{dBm}$.

beamformer that is based on realistic EH and energy consumption models at the relays. Our performance analysis results have illustrated that the proposed multi-relay scheme can deliver significant benefits as compared to single relay systems for a wide variety of relay placements. Finally, we set as a future objective the design of optimal relay selection policies for maximizing the average rate of our scheme.

\section{APPENDIX A}

\section{SOLVING OPTIMIZATION PROBLEM (P3)}

After dropping constraint $\gamma_{2} \in \mathbb{R}^{+}$, we can solve problem (P3) by separately considering the following two cases.

A. Case I: $D \leq A_{i}^{2}+C_{i}$

In this case (C3.1) is redundant. Moreover, from Theorem 2 we have that the optimal value is obtained when $\gamma_{2}^{2}=D-\gamma_{1}^{2}$. (P3) is therefore equivalently expressed as:

$$
\begin{array}{ll}
\underset{\gamma_{1}}{\operatorname{maximize}:} & \alpha \gamma_{1}+\beta \sqrt{D-\gamma_{1}^{2}}, \\
\text { subject to: } & \gamma_{1, \text { low }, i} \leq \gamma_{1} \leq \min \left\{\sqrt{D}, \gamma_{1, u p, i}\right\} .
\end{array}
$$

and the optimal $\gamma_{1}$ is easily obtained as:

$$
\gamma_{1}^{\star}=\sqrt{\frac{\alpha^{2} D}{\alpha^{2}+\beta^{2}}},
$$


provided that $\gamma_{1}{ }^{\star}$ respects $(\mathrm{C} 4.1)$. Otherwise the optimal solution is obtained as the value in the set $\left\{\gamma_{1, \text { low }, i}, \min \left\{\gamma_{1, \text { up }}, \sqrt{D}\right\}\right\}$ that maximizes the utility function in (P3). The optimal $\gamma_{2}$ is then $\gamma_{2}^{\star}=\sqrt{D-\gamma_{1}^{2}}$. Having investigated this first case, we now move to the investigation of the second one.

\section{B. Case II: $D \geq A_{i}^{2}+C_{i}$}

In this case both constraints (C3.1) and (C3.2) have to be taken into account. Employing then the result of Theorem 2 we discriminate the following two cases.

1) Solving problem (P3) with constraint (C3.2) being active: In this case, by setting $\gamma_{2}^{2}=D-\gamma_{1}^{2}$, after some straightforward manipulations we can rewrite problem (P3) in the equivalent form:

$$
\begin{aligned}
& \underset{\gamma_{1}}{\operatorname{maximize}}: \alpha \gamma_{1}+\beta \sqrt{D-\gamma_{1}^{2}} \\
& \text { subject to: }(\mathrm{C} 3.3), \gamma_{1} \in\left[\frac{\sqrt{D-C_{i}}-A_{i}}{B_{i}}, \sqrt{D}\right]
\end{aligned}
$$

Clearly, the solution to (P5) is the value $\gamma_{1}^{\star}$ in (40), provided that it is feasible. Otherwise, the solution to (P5) is selected among the values of the set $\left\{\max \left\{\gamma_{1, \text { low }, i}, \frac{\sqrt{D-C_{i}}-A_{i}}{B_{i}}\right\}, \min \left\{\gamma_{1, \text { up }, i}, \sqrt{D}\right\}\right\} \quad$ as the feasible one that results in the highest utility value. The value of $\gamma_{2}$ is then selected based on the fact that (C3.2) is active.

2) Solving problem (P3) with constraint (C3.1) being active: In this case we can set $\gamma_{2}^{2}=\left(A_{i}+B_{i} \gamma_{1}\right)^{2}+C_{i}-\gamma_{1}^{2}$. The constraint $\gamma_{1}^{2}+\gamma_{2}^{2} \leq D$ is then equivalent to the constraint $\left(A_{i}+B_{i} \gamma_{1}\right)^{2}+C_{i} \leq D$. The combination of this constraint with constraint $\gamma_{1} \in\left[\gamma_{1, \text { low }, i}, \gamma_{1, u p, i}\right]$ then leads to the following problem:

$$
\underset{\gamma_{\mathbf{1}}, \gamma_{\mathbf{2}}}{\operatorname{maximize}:} \alpha \gamma_{1}+\beta \sqrt{\left(A_{i}+B_{i} \gamma_{1}\right)^{2}+C_{i}-\gamma_{1}^{2}}
$$

subject to:

$$
\gamma_{1, \text { low }, i} \leq \gamma_{1} \leq \min \left\{\frac{\sqrt{D-C_{i}}-A_{i}}{B_{i}}, \sqrt{D}, \gamma_{1, u p, i}\right\} \text {. }
$$

To solve this problem, we start by finding the roots of the derivatives of its utility function, by solving the equation:

$$
\alpha=-\frac{\beta\left(A_{i} B_{i}+\left(B_{i}^{2}-1\right) \gamma_{1}\right)}{\sqrt{\left(A_{i}+B_{i} \gamma_{1}\right)^{2}+C_{i}-\gamma_{1}^{2}}} .
$$

Candidate roots for the above equation can be found by squaring both sides. We then obtain that roots of equation (43) need also be roots of the polynomial equation

$$
p_{2} \gamma_{1}^{2}+p_{1} \gamma_{1}+p_{0}=0
$$

where

$$
\begin{aligned}
& p_{2}=\left(B_{i}^{2}-1\right)\left(\beta^{2}\left(B_{i}^{2}-1\right)-\alpha^{2}\right) \\
& p_{1}=2 A_{i} B_{i}\left(\beta^{2}\left(B_{i}^{2}-1\right)-a^{2}\right) \\
& p_{0}=\beta^{2} A_{i}^{2} B_{i}^{2}-\alpha^{2}\left(A_{i}^{2}+C_{i}\right) .
\end{aligned}
$$

Note that due to the fact that the polynomial in (44) is of second degree, its solutions can be easily expressed in closed form. The solution to (P6) is then obtained by comparing the values for the utility function of problem (P6) achieved by the feasible solutions of (44), with the values achieved by the endpoints of constraint (C6.1) and selecting among these points the feasible solution that achieves the best value. The value of $\gamma_{2}$ is then determined by the the fact that (C3.1) is active. Having obtained the solutions for the case that constraints (C3.1) and (C3.2) are respectively active, we select the solution that results in maximizing the objective of (P3).

\section{REFERENCES}

[1] T. Riihonen, S. Werner, and R. Wichman, "Mitigation of loopback selfinterference in full-duplex MIMO relays," IEEE Transactions on Signal Processing, vol. 59, no. 12, pp. 5983-5993, Dec. 2011.

[2] S. Hu, Z. Ding, Q. Ni, W. Yu, and Z. Song, "Energy efficiency in energy harvesting cooperative networks with self-energy recycling," in IEEE 20th International Workshop on Computer Aided Modelling and Design of Communication Links and Networks (CAMAD), Sept 2015, pp. 59-63.

[3] S. Hu, Z. Ding, and Q. Ni, "Beamforming optimisation in energy harvesting cooperative full-duplex networks with self-energy recycling protocol," IET Communications, vol. 10, no. 7, pp. 848-853, 2016.

[4] O. T. Demir and T. E. Tuncer, "Optimum QoS-aware beamformer design for full-duplex relay with self-energy recycling," IEEE Wireless Commun. Lett., vol. 7, no. 1, pp. 122-125, Feb 2018.

[5] G. A. Ropokis, M. M. Butt, N. Marchetti, and L. A. DaSilva, "Optimal power allocation for energy recycling assisted cooperative communications," in IEEE Wireless Communications and Networking Conference Workshops (WCNCW), Mar. 2017, pp. 1-6.

[6] G. A. Ropokis, N. Marchetti, and L. A. DaSilva, "Cooperative beamforming exploiting energy recycling," in 2018 25th International Conference on Telecommunications (ICT), June 2018, pp. 577-582.

[7] O. T. Demir and T. E. Tuncer, "Robust optimum and near-optimum beamformers for decode-and-forward full-duplex multi-antenna relay with self-energy recycling," IEEE Trans. Wireless Comm., pp. 1-1, 2019.

[8] Y. Chen, N. Zhao, and M. Alouini, "Wireless energy harvesting using signals from multiple fading channels," IEEE Trans. Commun., vol. 65, no. 11, pp. 5027-5039, Nov 2017.

[9] Y. Zeng and R. Zhang, "Full-Duplex Wireless-Powered Relay With SelfEnergy Recycling," IEEE Wireless Commun. Lett., vol. 4, no. 2, pp. 201-204, Apr 2015.

[10] Y. Dong, M. J. Hossain, and J. Cheng, "Performance of wireless powered amplify and forward relaying over nakagami- $m$ fading channels with nonlinear energy harvester," IEEE Commun. Lett., vol. 20, no. 4, pp. 672-675, April 2016.

[11] P. N. Alevizos and A. Bletsas, "Sensitive and nonlinear far-field RF energy harvesting in wireless communications," IEEE Trans. Wireless Comm., vol. 17, no. 6, pp. 3670-3685, June 2018.

[12] X. Zhou, B. Bai, and W. Chen, "Greedy relay antenna selection for sum rate maximization in amplify-and-forward MIMO two-way relay channels under a holistic power model," IEEE Commun. Lett., vol. 19, no. 9, pp. 1648-1651, Sep. 2015.

[13] H. Liu, K. J. Kim, K. S. Kwak, and H. V. Poor, "Power splitting-based SWIPT with decode-and-forward full-duplex relaying," IEEE Trans. Wireless Comm., vol. 15, no. 11, pp. 7561-7577, Nov 2016.

[14] Y. Rajavi, M. Taghivand, K. Aggarwal, A. Ma, and A. S. Y. Poon, "An energy harvested ultra-low power transceiver for internet of medical things," in ESSCIRC Conference 2016: 42nd European Solid-State Circuits Conference, Sep. 2016, pp. 133-136.

[15] Y. Kim, H. S. Bhamra, J. Joseph, and P. P. Irazoqui, "An ultra-low-power RF energy-harvesting transceiver for multiple-node sensor application," IEEE Trans. Circuits Syst. II, Express Briefs, vol. 62, no. 11, pp. 10281032, Nov 2015. 